\title{
Whole Exome Sequencing Identifies Novel Genetic Alterations in Patients with Pheochromocytoma/ Paraganglioma
}

\author{
Soo Hyun Seo,*, Jung Hee $\mathrm{Kim}^{2}$,*, Man Jin $\mathrm{Kim}^{3}$, Sung Im $\mathrm{Cho}^{3}$, Su Jin $\mathrm{Kim}^{4}$, Hyein $\mathrm{Kang}^{4}$, Chan Soo Shin ${ }^{2}$, \\ Sung Sup Park ${ }^{3,5}$, Kyu Eun Lee ${ }^{4,5}$, Moon-Woo Seong ${ }^{3,5}$ \\ ${ }^{1}$ Department of Laboratory Medicine, Seoul National University Bundang Hospital, Seoul National University College of \\ Medicine, Seongnam; Departments of ${ }^{2}$ Internal Medicine, ${ }^{3}$ Laboratory Medicine, ${ }^{4}$ Surgery, Seoul National University Hospital, \\ Seoul National University College of Medicine; ${ }^{5}$ Cancer Research Institute, Seoul National University College of Medicine, \\ Seoul, Korea
}

Background: Pheochromocytoma and paragangliomas (PPGL) are known as tumors with the highest level of heritability, approximately $30 \%$ of all cases. Clinical practice guidelines of PPGL recommend genetic testing for germline variants in all patients. In this study, we used whole exome sequencing to identify novel causative variants associated with PPGL to improve the detection of rare genetic variants in our cohort.

Methods: Thirty-six tested negative for pathogenic variants in previous Sanger sequencing or targeted gene panel testing for PPGL underwent whole exome sequencing. Whole exome sequencing was performed using DNA samples enriched using TruSeq Custom Enrichment Kit and sequenced with MiSeq (Illumina Inc.). Sequencing alignment and variant calling were performed using SAMtools.

Results: Among previously mutation undetected 36 patients, two likely pathogenic variants and 13 variants of uncertain significance (VUS) were detected in 32 pheochromocytoma-related genes. SDHA c.778G >A (p.Gly260Arg) was detected in a patient with head and neck paraganglioma, and $K I F 1 B \mathrm{c} \cdot 2787-2 \mathrm{~A}>\mathrm{C}$ in a patient with a bladder paraganglioma. Additionally, a likely pathogenic variant in BRCA2, VUS in TP53, and VUS in NFU1 were detected.

Conclusion: Exome sequencing further identified genetic alterations by $5.6 \%$ in previously mutation undetected patients in PPGL. Implementation of targeted gene sequencing consisted of extended genes of PPGL in routine clinical screening can support the level of comprehensive patient assessment.

Keywords: Pheochromocytoma; Paraganglioma; Whole exome sequencing; Germ-line mutation; Molecular diagnostic techniques

Received: 6 July 2020, Revised: 8 October 2020, Accepted: 3 November 2020 Corresponding authors: Moon-Woo Seong

Department of Laboratory Medicine, Seoul National University Hospital, 101 Daehak-ro, Jongno-gu, Seoul 03080, Korea

Tel: +82-2-2072-4180, Fax: +82-2-747-0359, E-mail: MWSeong@snu.ac.kr

Kyu Eun Lee

Department of Surgery, Seoul National University Hospital, 101 Daehak-ro, Jongno-gu, Seoul 03080, Korea

Tel: +82-2-2072-0393, Fax: +82-2-741-6288, E-mail: kyueunlee@snu.ac.kr

\section{Copyright $(92020$ Korean Endocrine Society}

This is an Open Access article distributed under the terms of the Creative Commons Attribution Non-Commercial License (https://creativecommons.org/ licenses/by-nc/4.0/) which permits unrestricted non-commercial use, distribution, and reproduction in any medium, provided the original work is properly cited.

*These authors contributed equally to this work. 


\section{INTRODUCTION}

Pheochromocytoma and paragangliomas (PPGL) are the most heritable tumors, with around $30 \%$ of cases caused by pathogenic variants. More than 15 germline and 30 somatic variants of causative genes have been associated with the disease, demonstrating a high degree of heterogeneity [1-7].

Molecular PPGL subtypes can be classified into three groups according to the Cancer Genome Atlas [2]. One major cluster is the pseudohypoxic group, which includes $S D H x$ ( $S D H A, S D H B$, SDHC, SDHD), SDHAF2, FH, MDH2, IDH1, VHL, EPAS1, and $P H D 1 / 2$, with somatic and germline variants. Another cluster is the kinase signaling group, consisting of germline or somatic variants in RET, NF1, HRAS, MAX, and TMEM127. The third cluster is Wnt signaling group, which includes newly recognized somatic variants in CSDE1 as well as somatic gene fusions affecting MAML3.

Among those genes, RET, NF1, and $V H L$ are involved in three distinct clinical syndromes associated with PPGL: multiple endocrine neoplasia type 2 (MEN2) syndrome caused by $R E T$, neurofibromatosis type I caused by $N F 1$, and von HippelLindau disease caused by VHL. Aside from those three syndromes, germline variants in the succinate dehydrogenase (SDH) genes are the most common cause of PPGL, occurring in up to $25 \%$ of all PPGL patients [8]. Clinical practice guidelines of PPGL recommend testing for germline variants in all patients by accredited laboratories $[9,10]$. Clinical characteristics of PPGL can be classified according to genetic clusters, which can lead to different follow-up tests and treatments guided by the underlying molecular cause. However, some patients may not express causative genes included in the molecular subtypes, thus remaining unclassified.

In this study, whole exome sequencing (WES) was used to screen for novel causative variants associated with PPGL to improve the detection rate of rare genetic variants in our cohort. Additional screening for variants in other genes related to cancerous disease or mitochondrial function was also performed.

\section{METHODS}

\section{Subjects}

Among patients diagnosed with PPGLs at the Seoul National University Hospital, 36 were recruited due to high risk of genetic diseases: metastasis $(n=9)$, bilateral diseases $(n=2)$, paraganglioma ( $n=16)$, aged under 35 years $(n=9)$. Among them, 20 patients were negative for $S D H B, S D H D, V H L$, and RET genes using Sanger sequencing and multiplex ligation-dependent probe amplification before March 2014. In March 2014, the targeted next-generation sequencing panel for PPGL was developed and used to test the additional 16 patients. All 16 patients were negative for MAX, NF1, RET, SDHA, SDHAF2, SDHB, $S D H C, S D H D, T M E M 127$, and $V H L$. This study was approved by the Institutional Review Board of Seoul National University Hospital (IRB No. 2004-012-1115). Informed consent from all patients was obtained.

PPGL was diagnosed by elevated levels of catecholamine and/or histological confirmation after surgery. We conducted serum fractionated metanephrine or 24-hour urine catecholamine/fractionated metanephrine. Hormone type was classified as epinephrine, norepinephrine, or nonfunctioning [11]. Elevated metanephrine with or without high normetanephrine levels was designated as epinephrine type. Elevated normetanephrine levels without high metanephrine levels were considered as norepinephrine type. Nonfunctioning type indicated a normal range of fractionated metanephrine levels.

Thoraco-abdominal computed tomography (CT) or magnetic resonance imaging was performed for anatomical localization. ${ }^{123}$ I-metaiodobenzylguanidine (MIBG), positron emission tomography/CT with ${ }^{68} \mathrm{Ga}$-labeled DOTA ${ }^{0}-\mathrm{Tyr}^{3}$ octreotide (DOTATOC) or ${ }^{18} \mathrm{~F}$-labeled fluorodeoxyglucose (FDG) was conducted to detect multifocal lesions or metastasis. Metastasis was defined as the presence of PPGL tumors in non-chromaffin organs at diagnosis or during follow-up [12].

\section{Molecular genetic testing}

DNA was extracted from whole blood samples obtained from 36 patients. WES was performed using DNA samples enriched using TruSeq Custom Enrichment Kit and sequenced with MiSeq (Illumina Inc., San Diego, CA, USA). Sequencing alignment and variant calling were performed using SAMtools. Copy number variation (CNV) analysis for the genes included in the panel was not performed.

\section{Variant filtering and interpretation of clinical significance} Exonic variants with nonsynonymous variants and intronic variants within $10 \mathrm{bp}$ from the exonic region were included. Allele frequencies in normal controls (gnomeAD) and in silico prediction results were considered (SIFT, PolyPhen2, and MutationTaster). The highest minor allele frequency (MAF) in the patient population was taken into consideration, and variants that had MAF $>0.1 \%$ were filtered out. Classification of each retained variant was performed according to the American College of 
Medical Genetics and Genomics and the Association for Molecular Pathology (ACMG-AMP) 2015 guidelines [13]. For previously reported variants, segregation and functional test results were reviewed. Variants were screened for the 32 pheochromocytoma-related genes (ATRX, BRAF, CDKN2A, DLST, DNMT3A, EGLN1, EGLN2, EPAS1, FGFR1, FH, GOT2, H3F3A, HRAS, IDH1, IDH2, IDH3B, KIF1B, KMT2D, MAX, MDH2, MERTK, MET, NF1, RET, SDHA, SDHAF2, SDHB, SDHC, SDHD, SLC25A11, TMEM127, and VHL). Genes related to other types of cancerous disease or encoding for mitochondria-localized proteins were screened for additional variants.

\section{RESULTS}

\section{Patients' characteristics}

Among the 36 patients included in this study, 19 were female and 17 were male. The mean age at the time of diagnosis was 40.2 (range, 12 to 85) (Table 1). Seventeen patients were diagnosed with pheochromocytoma, four bilateral and two multifocal, while 19 patients were diagnosed with paraganglioma. None of the patients had a family history of pheochromocytoma or paraganglioma. Nine patients presented metastatic lesions, and three patients showed relapse during the follow-up period.

\section{Identification of germline variants in pheochromocytoma- related genes}

Average coverage depth in target regions of the whole exome panel was $80.7 \mathrm{X} ; 99.1 \%$ of the bases had coverage of $\geq 20 \mathrm{X}$, which was the minimal level of acceptable coverage considered. Among 36 patients, 14 patients were found to carry at least one variant of interest (VOI) in 32 pheochromocytoma-related genes. A total of 15 VOIs were detected, two were classified as likely pathogenic variants and 13 as variants of uncertain significance (VUS) (Table 2). SDHA c.778G $>$ A (p.Gly260Arg) was detected in a patient negative for $S D H B, S D H D, V H L$, and RET genes. This was a previously reported variant in paraganglioma [14-16], known to be a loss-of-function variant according to functional studies [15]. KIF1B c.2787-2A >C, a likely pathogenic variant that had not been previously reported, was detected in a patient with a bladder paraganglioma. Other variants detected in pheochromocytoma-related genes lacked strong supporting evidence for pathogenic classification. $S D H C$ c.478G $>$ A (p.Val160Met) has not been reported previously, but other missense variants near this amino acid residue such as p. Leu158Pro and p. Leu161Val had been detected in PPGL patients [17-19]. FH c.418G $>\mathrm{C}$ (p.Val140Leu) had been submitted in ClinVar as a VUS, but
c.419T > G (p. Val140Gly) involving the same amino acid residue has been reported in leiomyomatosis and renal cell cancer patients [20]. In addition, a novel nonsense variant, c.914G $>$ A (p.Trp305*) in DNMT3A, showed variant allele frequency of $18 \%$ in exome sequencing data, and subsequent validation by Sanger sequencing showed a small alternate peak in the region (Supplemental Fig. S1).

\section{Identification of germline variants in other genes}

Also, we screened for germline variants in other cancer-related genes or mitochondria-related genes. One likely pathogenic variant in BRCA2 and one VUS in TP53 were detected in cancer-related genes (Table 2). A BRCA2 splice-site variant, c.8488$1 \mathrm{G}>\mathrm{A}$, was detected in a 25 -year-old male patient with earlyonset paraganglioma. He had no personal cancer history, nor a family history of cancer related to $B R C A 2$. A patient with VUS in TP53, c.566C > T (p.Ala189Val), had previously been diagnosed with breast cancer, endometrial polyp and also had a brother who had been diagnosed with choriocarcinoma. Additionally, we found a missense VUS c.473G > A in NFU1, which is a causative gene of multiple mitochondrial dysfunctions syndrome 1 (MMDS1).

\section{DISCUSSION}

Among the 36 patients found to be negative for routine clinical gene testing, only two were found to be positive for likely pathogenic variants $(2 / 36=5.6 \%) . S D H A$ c. $778 \mathrm{G}>\mathrm{A}$ (p.Gly260Arg) was shown to be a loss-of-function variant in functional studies in a yeast strain lacking Sdh1 [15]. Pathogenic germline SDHA variants were previously identified in $7.6 \%$ of patients with PGL, with diagnosis occurring at a significantly younger age in patients carrying the SDHA variants [21]. The patient carrying the likely pathogenic $S D H A$ variant in this study was diagnosed with head and neck paraganglioma at the age of 20 and was the second youngest patient of our study cohort. Missense variants in the $K I F 1 B$ gene had been previously detected in samples of pheochromocytoma [22,23], along with a splice site variant [24]. Yet, no previous reports of paraganglioma with a pathogenic $K I F 1 B$ variant have been published. Our patient carrying a $K I F 1 B$ c. $2787-2 \mathrm{~A}>\mathrm{C}$ had a bladder paraganglioma, which may be the first paraganglioma to be reported carrying a $K I F 1 B$ variant. The overall positive rate of pathogenic variants in the whole cohort of the apparently sporadic PPGL in our institution was $21.7 \%$ (35 among 161 PPGL patients). The most commonly mutated gene was RET (31.4\%), followed by VHL (25.7\%), 


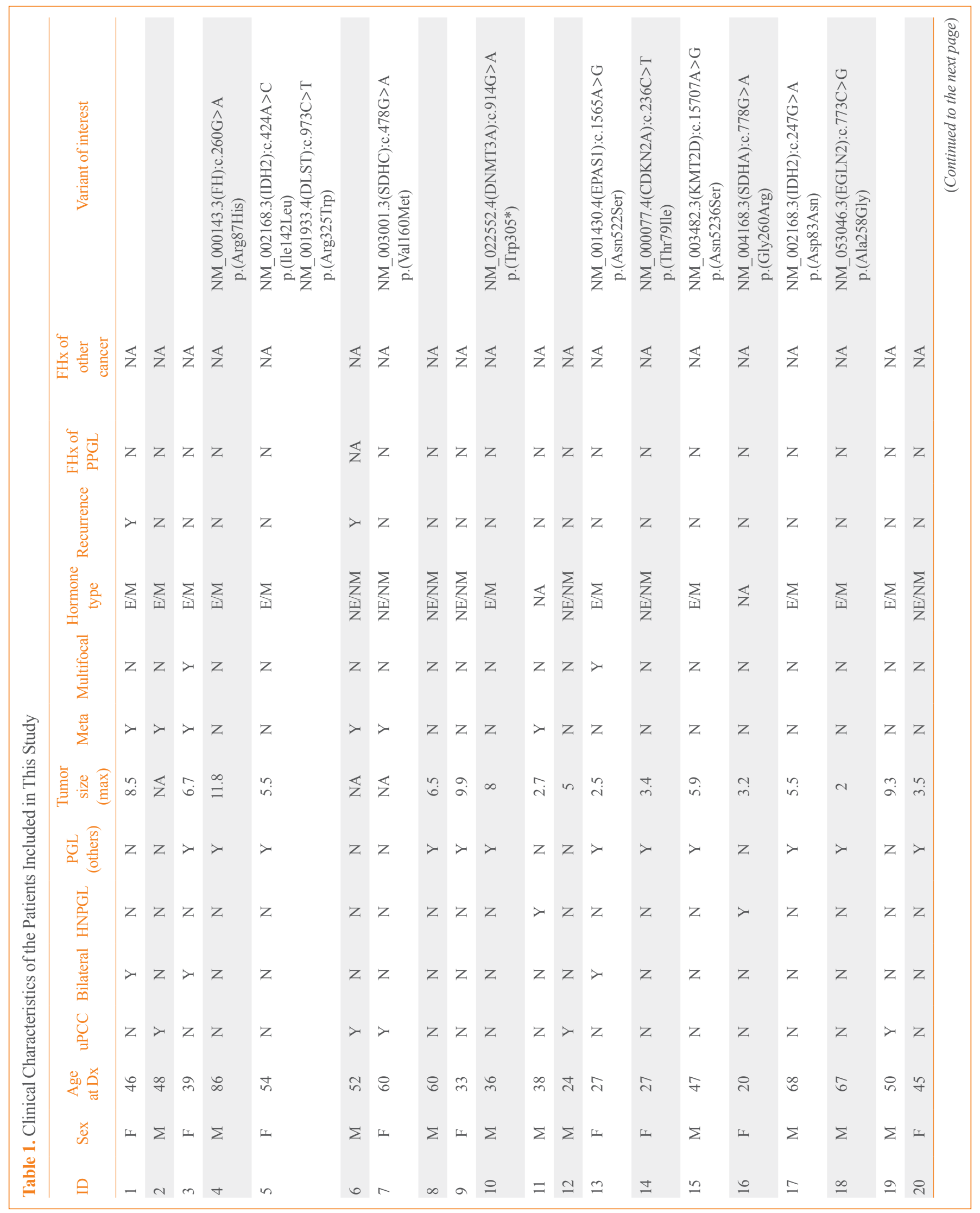




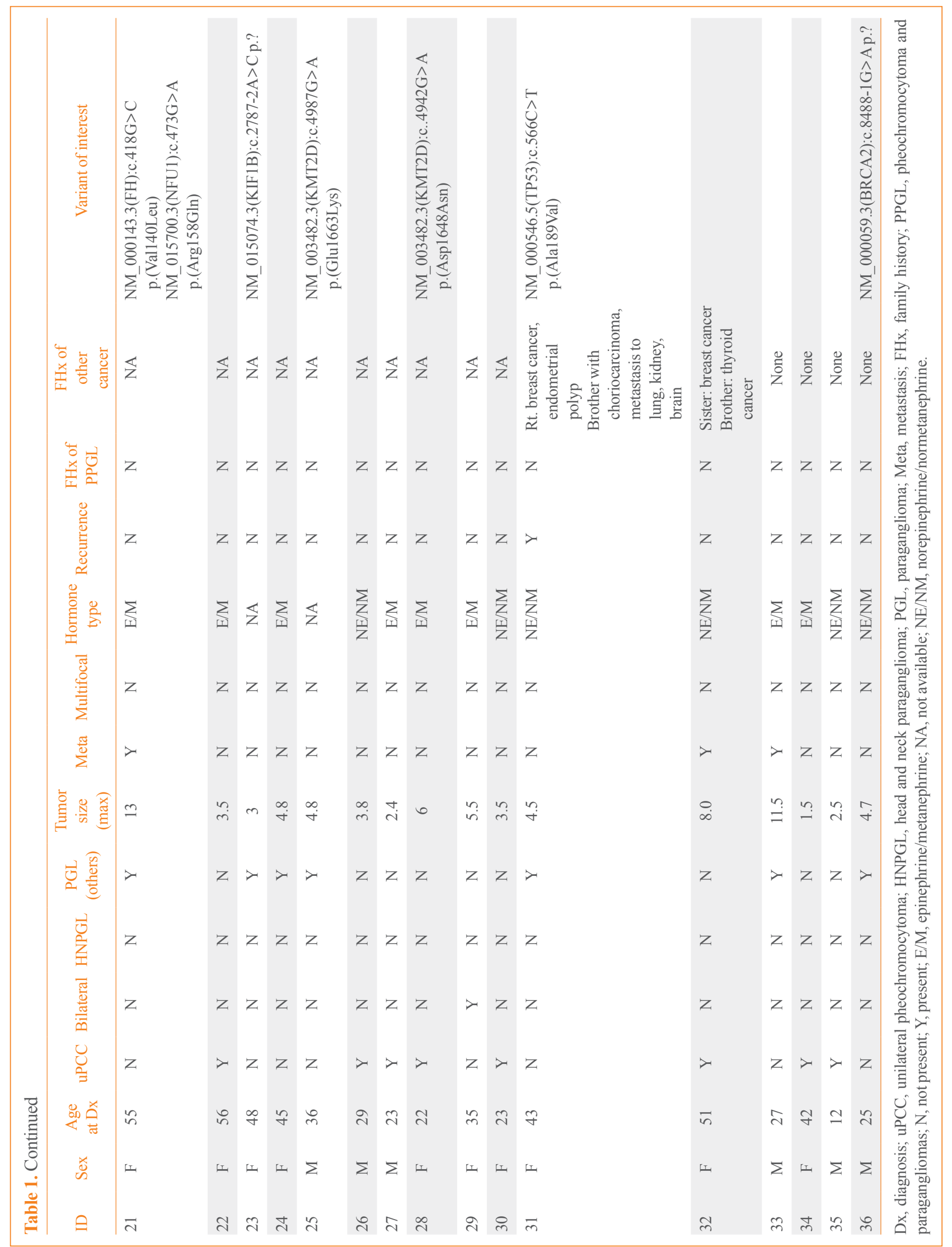




\begin{tabular}{|c|c|c|c|c|c|c|c|}
\hline 23 & KIFIB & NM_015074 & c. $2787-2 \mathrm{~A}>\mathrm{C}$ & p.? & $-/-/ \mathrm{D}$ & - & LP \\
\hline 16 & SDHA & NM_004168 & c. $778 \mathrm{G}>\mathrm{A}$ & p.Gly260Arg & $\mathrm{D} / \mathrm{D} / \mathrm{D}$ & - & LP [14-16] \\
\hline $5^{\mathrm{b}}$ & $D L S T$ & NM_001933 & c. $973 \mathrm{C}>\mathrm{T}$ & p.Arg325Trp & $\mathrm{D} / \mathrm{D} / \mathrm{D}$ & AFR $0.012 \%$ & VUS \\
\hline 10 & DNMT3A & NM_175629 & c. $914 \mathrm{G}>\mathrm{A}^{\mathrm{c}}$ & p.Trp305 & $-/-/ \mathrm{D}$ & AFR $0.0062 \%$ & VUS \\
\hline 18 & EGLN2 & NM_053046 & c. $773 \mathrm{C}>\mathrm{G}$ & p.Ala258Gly & $\mathrm{T} / \mathrm{B} / \mathrm{D}$ & EAS $0.011 \%$ & VUS \\
\hline 13 & EPAS1 & NM_001430 & c. $1565 \mathrm{~A}>\mathrm{G}$ & p.Asn522Ser & $\mathrm{T} / \mathrm{B} / \mathrm{N}$ & EAS $0.033 \%$ & VUS \\
\hline 4 & $F H$ & NM_000143 & c. $260 \mathrm{G}>\mathrm{A}$ & p.Arg87His & $\mathrm{D} / \mathrm{D} / \mathrm{D}$ & EAS $0.033 \%$ & VUS \\
\hline 28 & $K M T 2 D$ & NM_003482 & c. $4942 \mathrm{G}>\mathrm{A}$ & p.Asp1648Asn & $\mathrm{D} / \mathrm{P} / \mathrm{D}$ & EAS $0.015 \%$ & VUS \\
\hline 25 & $K M T 2 D$ & NM_003482 & c. $4987 \mathrm{G}>\mathrm{A}$ & p.Glu1663Lys & $\mathrm{D} / \mathrm{D} / \mathrm{D}$ & NFE $0.001 \%$ & VUS \\
\hline 15 & $K M T 2 D$ & NM_003482 & c. $15707 \mathrm{~A}>\mathrm{G}$ & p.Asn5236Ser & $\mathrm{T} / \mathrm{P} / \mathrm{D}$ & EAS $0.006 \%$ & VUS \\
\hline 7 & $S D H C$ & NM_003001 & c. $478 \mathrm{G}>\mathrm{A}$ & p.Val160Met & $\mathrm{D} / \mathrm{P} / \mathrm{D}$ & - & VUS \\
\hline 36 & $B R C A 2$ & NM_000059 & c. $8488-1 \mathrm{G}>\mathrm{A}$ & & $-/-/ \mathrm{D}$ & - & $\begin{array}{l}\text { Pathogenic for breast/ } \\
\text { ovarian cancer }\end{array}$ \\
\hline $21^{\mathrm{b}}$ & NFU1 & NM_015700 & c. $473 \mathrm{G}>\mathrm{A}$ & p.Arg158Gln & $\mathrm{D} / \mathrm{D} / \mathrm{D}$ & EAS $0.0054 \%$ & VUS \\
\hline 31 & TP53 & NM_000546 & c. $566 \mathrm{C}>\mathrm{T}$ & p.Ala189Val & $\mathrm{D} / \mathrm{D} / \mathrm{D}$ & EAS $0.027 \%$ & VUS \\
\hline \multicolumn{8}{|c|}{ 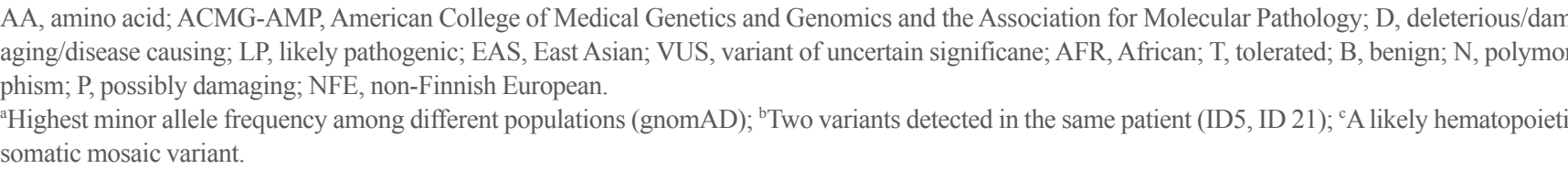 } \\
\hline
\end{tabular}

SDHB (17.1\%), and SDHD (14.3\%) (unpublished data). Positive rate for $S D H A$ was $2.9 \%$, though the result seems underestimated since SDHA has been included in the panel recently. Our data showed targeted gene panel with extended genes related to PPGL would benefit by increasing the positive rate of pathogenic variants.

Among the VUSs, a novel nonsense variant, c. $914 \mathrm{G}>\mathrm{A}$ (p.Trp305*) was detected in DNMT3A. This variant may be a likely hematopoietic somatic mosaic variant unrelated to the paraganglioma. Germline variants of DNMT3A previously reported in paraganglioma had been gain-of-function missense variants [25], while most of the likely hematopoietic somatic mosaic variants detected in multiple cancers were loss-of-function variants [26].

In other cancer-related genes, $B R C A 2$ c. $8488-1 \mathrm{G}>\mathrm{A}$, detected in a 25 -year-old male patient with early-onset paraganglioma. The patient had no personal history or family history of
$B R C A 2$ related cancer. Germline $B R C A 1 / 2$ variants, most commonly associated genes in familial breast and ovarian cancer, are also known to be associated with other cancers such as prostate, colon, gastric, pancreatic cancer. BRCA1/2 variants are not regarded as genetic causes for adrenal tumors, but there had been a previously reported case of pheochromocytoma who carried $B R C A 2$ variants [27]. A 40-year-old Ashkenazi woman was diagnosed with pheochromocytoma, and later diagnosed with infiltrating ductal carcinoma at 61 years of age. The patient carrying a $B R C A 2$ splicing variant in our study had been diagnosed with paraganglioma at the age of 25 , and yet he had not been diagnosed with additional cancer until now. However, at 15 months of age, the patient underwent a Fontan operation [28]. Thus, hypoxic condition may be the second hit for development for paraganglioma. Although the causative role of this variant for the diagnosis of pheochromocytoma cannot be proven, the $B R C A 2$ germline variants may be associated with an increased 
risk for adrenal tumors. Another missense VUS, c.473G $>$ A (p.Arg158Gln) in NFU1, was found in an individual who also carried a missense variant c.418G $>\mathrm{C}$ (p. Val140Leu) in $F H$. NFU1 is an essential iron-sulfur $(\mathrm{Fe} / \mathrm{S})$ protein implicated in multiple metabolic pathways and energy production, and acts as a maturation factor of respiratory complex II (SDH) [29]. Though this gene is known to be associated with MMDS1, which is inherited in an autosomal recessive pattern, a variant that affects the function of the protein may be involved with compromised SDH function [30,31]. Association of the disease and the BRCA2 variant, as well as VUS detected in other genes, should be assessed in further studies.

This study has several limitations. Though VUS reclassification is considered important as the genetic testings are becoming more available [32], familial screening was not performed in any of the patients. Segregation data would have provided more evidence that can lead to the reclassification of numerous VUS detected. Also, gross defects such as CNV were excluded, only analyzing single nucleotide variants or small insertion/deletions. Moreover, somatic variants in the tissue were not analyzed though it would have explained the additional driver alteration of the disease other than the germline portion. Further evaluation regarding family testing, $\mathrm{CNV}$ analysis, and sequencing of the tissue samples would improve the overall detection rate of the causative genetic variants.

In conclusion, we analyzed the WES data of PPGL patients with no causative genetic variant detected in routine clinical gene testing. Likely pathogenic variants were detected in two patients, which led to a $5.6 \%$ increase in molecularly confirmed PPGL patients. While implementation of WES for detection of germline variants in PPGL patients has not yet been widely adopted in clinical laboratories, implementation of targeted gene sequencing consisted of extended genes of PPGL in routine clinical screening can support the level of comprehensive patient assessment.

\section{CONFLICTS OF INTEREST}

No potential conflict of interest relevant to this article was reported.

\section{ACKNOWLEDGMENTS}

This study was supported by the Basic Science Research Program through the National Research Foundation of Korea by the grant NRF-2020R1A2C1014419.

\section{AUTHOR CONTRIBUTIONS}

Conception or design: S.H.S., J.H.K., M.W.S. Acquisition, analysis, or interpretation of data: S.H.S., J.H.K., M.J.K., S.I.C., S.J.K., H.K., C.S.S., S.S.P., K.E.L., M.W.S. Drafting the work or revising: S.H.S., J.H.K., K.E.L., M.W.S. Final approval of the manuscript: K.E.L., M.W.S.

\section{ORCID}

Soo Hyun Seo https://orcid.org/0000-0002-6899-4967

Jung Hee Kim https://orcid.org/0000-0003-1932-0234

Kyu Eun Lee https://orcid.org/0000-0002-2354-3599

Moon-Woo Seong https://orcid.org/0000-0003-2954-3677

\section{REFERENCES}

1. Pillai S, Gopalan V, Smith RA, Lam AK. Updates on the genetics and the clinical impacts on phaeochromocytoma and paraganglioma in the new era. Crit Rev Oncol Hematol 2016;100:190-208.

2. Crona J, Tajeb D, Pacak K. New perspectives on pheochromocytoma and paraganglioma: toward a molecular classification. Endocr Rev 2017;38:489-515.

3. Toledo RA. Genetics of pheochromocytomas and paragangliomas: an overview on the recently implicated genes MERTK, MET, fibroblast growth factor receptor 1, and H3F3A. Endocrinol Metab Clin North Am 2017;46:459-89.

4. Alrezk R, Suarez A, Tena I, Pacak K. Update of pheochromocytoma syndromes: genetics, biochemical evaluation, and imaging. Front Endocrinol (Lausanne) 2018;9:515.

5. Koopman K, Gaal J, de Krijger RR. Pheochromocytomas and paragangliomas: new developments with regard to classification, genetics, and cell of origin. Cancers (Basel) 2019; 11:1070.

6. Neumann HP, Young WF Jr, Eng C. Pheochromocytoma and paraganglioma. N Engl J Med 2019;381:552-65.

7. Antonio K, Valdez MM, Mercado-Asis L, Tajeb D, Pacak K. Pheochromocytoma/paraganglioma: recent updates in genetics, biochemistry, immunohistochemistry, metabolomics, imaging and therapeutic options. Gland Surg 2020;9:10523.

8. Benn DE, Robinson BG, Clifton-Bligh RJ. 15 Years of paraganglioma: clinical manifestations of paraganglioma syndromes types 1-5. Endocr Relat Cancer 2015;22:T91-103.

9. Lenders JW, Duh QY, Eisenhofer G, Gimenez-Roqueplo 
AP, Grebe SK, Murad MH, et al. Pheochromocytoma and paraganglioma: an Endocrine Society Clinical Practice Guideline. J Clin Endocrinol Metab 2014;99:1915-42.

10. Nolting S, Ullrich M, Pietzsch J, Ziegler CG, Eisenhofer G, Grossman A, et al. Current management of pheochromocytoma/paraganglioma: a guide for the practicing clinician in the era of precision medicine. Cancers (Basel) 2019;11:1505.

11. Kimura N, Takayanagi R, Takizawa N, Itagaki E, Katabami T, Kakoi N, et al. Pathological grading for predicting metastasis in phaeochromocytoma and paraganglioma. Endocr Relat Cancer 2014;21:405-14.

12. Thompson LD. Pheochromocytoma of the Adrenal gland Scaled Score (PASS) to separate benign from malignant neoplasms: a clinicopathologic and immunophenotypic study of 100 cases. Am J Surg Pathol 2002;26:551-66.

13. Richards S, Aziz N, Bale S, Bick D, Das S, Gastier-Foster J, et al. Standards and guidelines for the interpretation of sequence variants: a joint consensus recommendation of the American College of Medical Genetics and Genomics and the Association for Molecular Pathology. Genet Med 2015; 17:405-24.

14. Evenepoel L, Papathomas TG, Krol N, Korpershoek E, de Krijger RR, Persu A, et al. Toward an improved definition of the genetic and tumor spectrum associated with SDH germ-line mutations. Genet Med 2015;17:610-20.

15. Bannon AE, Kent J, Forquer I, Town A, Klug LR, McCann $\mathrm{K}$, et al. Biochemical, molecular, and clinical characterization of succinate dehydrogenase subunit a variants of unknown significance. Clin Cancer Res 2017;23:6733-43.

16. Bausch B, Schiavi F, Ni Y, Welander J, Patocs A, Ngeow J, et al. Clinical characterization of the pheochromocytoma and paraganglioma susceptibility genes SDHA, TMEM127, MAX, and SDHAF2 for gene-informed prevention. JAMA Oncol 2017;3:1204-12.

17. Lefebvre S, Borson-Chazot F, Boutry-Kryza N, Wion N, Schillo F, Peix JL, et al. Screening of mutations in genes that predispose to hereditary paragangliomas and pheochromocytomas. Horm Metab Res 2012;44:334-8.

18. Ben Aim L, Pigny P, Castro-Vega LJ, Buffet A, Amar L, Bertherat J, et al. Targeted next-generation sequencing detects rare genetic events in pheochromocytoma and paraganglioma. J Med Genet 2019;56:513-20.

19. Bauters C, Vantyghem MC, Leteurtre E, Odou MF, Mouton C, Porchet N, et al. Hereditary phaeochromocytomas and paragangliomas: a study of five susceptibility genes. J Med Genet 2003;40:e75.
20. Espana A, Ornilla E, Zarate R, Gil-Bazo I, Briones P. Myopathy in hereditary leiomyomatosis and renal cell cancer in an extended family. Br J Dermatol 2015;172:1442-5.

21. Van der Tuin K, Mensenkamp AR, Tops CM, Corssmit EP, Dinjens WN, van de Horst-Schrivers AN, et al. Clinical aspects of SDHA-related pheochromocytoma and paraganglioma: a nationwide study. J Clin Endocrinol Metab 2018; 103:438-45.

22. Schlisio S, Kenchappa RS, Vredeveld LC, George RE, Stewart R, Greulich H, et al. The kinesin KIF1Bbeta acts downstream from EgIN3 to induce apoptosis and is a potential 1p36 tumor suppressor. Genes Dev 2008;22:884-93.

23. Welander J, Andreasson A, Juhlin CC, Wiseman RW, Backdahl M, Hoog A, et al. Rare germline mutations identified by targeted next-generation sequencing of susceptibility genes in pheochromocytoma and paraganglioma. J Clin Endocrinol Metab 2014;99:E1352-60.

24. Pillai S, Gopalan V, Lo CY, Liew V, Smith RA, Lam AK. Silent genetic alterations identified by targeted next-generation sequencing in pheochromocytoma/paraganglioma: a clinicopathological correlations. Exp Mol Pathol 2017;102: 41-6.

25. Remacha L, Curras-Freixes M, Torres-Ruiz R, Schiavi F, Torres-Perez R, Calsina B, et al. Gain-of-function mutations in DNMT3A in patients with paraganglioma. Genet Med 2018;20:1644-51.

26. Schrader KA, Cheng DT, Joseph V, Prasad M, Walsh M, Zehir A, et al. Germline variants in targeted tumor sequencing using matched normal DNA. JAMA Oncol 2016;2:104-11.

27. Barak F, Shiri-Svredlov R, Bruchim-Bar Sade R, Kruglikova A, Friedman E, Ben-Dor D, et al. Adrenal tumors in BRCA1/ BRCA2 mutation carriers. Am J Med Genet 2001;98:277-9.

28. Song MK, Kim GB, Bae EJ, Lee YA, Kim HY, Min SK, et al. Pheochromocytoma and paraganglioma in Fontan patients: common more than expected. Congenit Heart Dis 2018;13:608-16.

29. Wesley NA, Wachnowsky C, Fidai I, Cowan JA. Understanding the molecular basis for multiple mitochondrial dysfunctions syndrome 1 (MMDS1): impact of a disease-causing Gly189Arg substitution on NFU1. FEBS J 2017;284:383848.

30. Lill R, Hoffmann B, Molik S, Pierik AJ, Rietzschel N, Stehling $\mathrm{O}$, et al. The role of mitochondria in cellular iron-sulfur protein biogenesis and iron metabolism. Biochim Biophys Acta 2012;1823:1491-508.

31. Van Vranken JG, Na U, Winge DR, Rutter J. Protein-medi- 
ated assembly of succinate dehydrogenase and its cofactors. Crit Rev Biochem Mol Biol 2015;50:168-80.
32. Fatkin D, Johnson R. Variants of uncertain significance and “missing pathogenicity”. J Am Heart Assoc 2020;9:e015588. 